\title{
Development of acute myocardial infarction in a young female patient with essential thrombocythemia treated with anagrelide: a case report
}

\author{
Young-Hyo Lim ${ }^{1}$, Young Yiul Lee ${ }^{2}$, Jae Hoon Kim ${ }^{1}$, Jinho Shin ${ }^{1}$, Jae Ung Lee ${ }^{1}$, Kyung-Soo Kim ${ }^{1}$, \\ Soon-Kil Kim ${ }^{1}$, Jeong Hyun Kim ${ }^{1}$, Heon Kil Lim ${ }^{1}$ \\ ${ }^{1}$ Division of Cardiology, ${ }^{2}$ Division of Hematology/Oncology, Department of Internal Medicine, College of Medicine, Hanyang \\ University, Seoul, Korea
}

\author{
p-ISSN 1738-7949 / e-ISSN 2092-9129 \\ DOI: $10.5045 / \mathrm{kjh} .2010 .45 .2 .136$ \\ Korean J Hematol 2010;45:136-8. \\ Received on March 24, 2010 \\ Revised on May, 19, 2010 \\ Accepted on May, 24, 2010

\section{Correspondence to} \\ Young Yiul Lee, M.D., Ph.D. \\ Division of Hematology/Oncology, \\ Department of Internal Medicine, \\ Hanyang University Hospital, 17, Haeng \\ Dang-dong, Sung Dong-gu, Seoul 133-792, \\ Korea \\ Tel: $+82-2-290-8334$ \\ Fax: +82-2-298-9183 \\ E-mail: leeyy@hanyang.ac.kr
}

(C) 2010 Korean Society of Hematology
Essential thrombocythemia (ET) is a chronic myeloproliferative disorder with a prolonged clinical course. Since this disorder is considered to be at increased risk of thromboembolism, therapy is mainly focused on the decreased risk of thrombohemorrhagic events by use of cytotoxic agents. Anagrelide is a phosphodiesterase III inhibitor which is utilized in the treatment of ET for the reduction of platelets. However, patients treated with anagrelide might experience cardiovascular adverse effects including myocardial infarction $(\mathrm{MI})$, although these events are rare. Herein, we report a case of a 30-year-old female with well controlled ET by anagrelide, who eventually developed an acute non-ST elevation myocardial infarction (MI). There has no found any cardiovascular risk factors in this ET patient, strongly suggesting that anagrelide might be the cause of MI. Therefore, cardiovascular function should be monitored in those patients prescribed with anagrelide.

Key Words Anagrelide, Essential thrombocythemia, Acute myocardial infarction

\section{INTRODUCTION}

Essential thrombocythemia (ET), an acquired myeloproliferative neoplasm, is characterized by excessive proliferation of megakaryocytes accompanied by persistent peripheral thrombocytosis [1]. Clinically, patients with ET develop thrombosis, hemorrhage, and microvascular complications. An increased number of circulating platelets is known to increase the risk of progression of these complications, as well as predispose the subject to thrombotic events. The main treatment strategy for ET is to rapidly decrease the patient's platelet count to a normal level in order to prevent the development of ET-related complications. In addition, ideal therapeutic agents should have limited side effects and minimal long-term safety concerns. A number of therapeutic agents have been studied for the treatment of ET, including alkylating agents, hydroxyurea, and interferon- $\alpha[2,3]$. Recently, anagrelide has been introduced as a treatment for
ET. Anagrelide is an oral imidazoquinazoline agent with a selective effect on the megakaryocyte cell lineage through the inhibition of cyclic-AMP phosphodiesterase III activity, which inhibits platelet aggregation $[4,5]$. Use of anagrelide effectively decreases the incidence of thromboembolic and hemorrhagic events. However, it can also directly induce vasospasm of the coronary arteries, and can lead to other serious cardiovascular side effects, including congestive heart failure, arrhythmias, and acute coronary syndrome [6]. Acute coronary syndromes are reported in $1-5 \%$ of patients treated with anagrelide, although the overall incidence of acute coronary syndromes is very rare [4, 7-9]. Here, we describe the case of a 30-year-old woman with ET who developed an acute, non-ST-segment elevation myocardial infarction (MI) with severe chest pain following the use of anagrelide. This patient had no cardiovascular risk factors, suggesting that anagrelide may have been the cause of her acute MI.

\footnotetext{
This is an Open Access article distributed under the terms of the Creative Commons Attribution Non-Commercial License (http://creativecommons.org/licenses/by-nc/3.0)
} which permits unrestricted non-commercial use, distribution, and reproduction in any medium, provided the original work is properly cited. 


\section{CASE REPORT}

A 30-year-old female patient presented to the emergency department with dyspnea on exertion and severe left anterior chest pain that had begun about 30 minutes prior to arrival, and which increased in intensity over time. She reported a history of exertional chest pain that had begun 10 days prior to her presentation. Previous studies, including electrocardiography (ECG), cardiac biomarkers, echocardiography, and an exercise treadmill test had shown no abnormalities. Her chest pain had been slightly alleviated by sublingual nitroglycerine. The patient had no smoking history and had no cardiovascular risk factors such as hypertension, dyslipidemia, or diabetes mellitus. The patient had been diagnosed with ET 6 months previously at a workplace health screening, and was being treated with $100 \mathrm{mg}$ aspirin, $500 \mathrm{mg}$ hydroxyurea twice a day, and $1 \mathrm{mg}$ anagrelide three times daily. Her ET was relatively well controlled without any other complications, and her platelet counts had been within the normal range. Initial vital signs were stable, with a blood pressure of $110 / 80 \mathrm{mmHg}$, a heart rate of 78 beats/min, and a respiratory rate of 18 breaths $/ \mathrm{min} . \mathrm{S}_{1}$ and $\mathrm{S}_{2}$ were normal and $\mathrm{S}_{4}$ was faintly audible at the apex. An ECG showed T-wave inversions in leads $\mathrm{I}$, aVL, and in $\mathrm{V}_{2}$ through

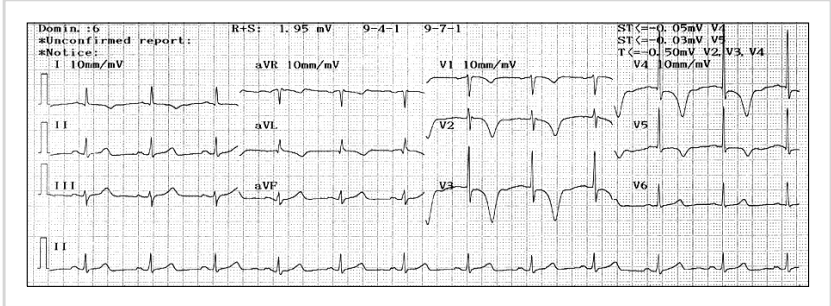

Fig. 1. Initial electrocardiograph showing T-wave inversions in leads I, $\mathrm{aVL}$, and $\mathrm{V}_{2}$ through $\mathrm{V}_{5}$, which are consistent with myocardial ischemia.
$\mathrm{V}_{5}$, which were consistent with myocardial ischemia (Fig. 1). Neither cardiomegaly nor pulmonary congestion was noted on a chest X-ray. The MB fraction of creatinine phosphokinase (CK-MB) was $8.5 \mathrm{ng} / \mathrm{mL}$ and cardiac troponin I was $3.08 \mathrm{ng} / \mathrm{mL}$ (upper normal limit, $0.07 \mathrm{ng} / \mathrm{mL}$ ). B-type natriuretic peptide $(100 \mathrm{pg} / \mathrm{mL})$ was also slightly elevated. A complete blood count showed the following: a leukocyte count of $3.70 \times 10^{3} / \mu \mathrm{L}$, hemoglobin of $10.5 \mathrm{~g} / \mathrm{dL}$, and a platelet count of $388 \times 10^{3} / \mu \mathrm{L}$. Low density lipoprotein (LDL) cholesterol level was $54 \mathrm{mg} / \mathrm{dL}$. An echocardiograph revealed hypokinesis in the anteroseptal wall and a left ventricular ejection fraction of $55 \%$. These results were compatible with an acute, non-ST-elevation MI. Immediately, the patient was treated with anti-platelet therapy (300 mg aspirin and $600 \mathrm{mg}$ clopidogrel). In addition, according to the early invasive strategy, coronary angiography was performed, which revealed significant segmental stenosis (90\%) of the mid left anterior descending artery (LAD) (Fig. 2A). The segmental lesion in the mid LAD did not respond to intracoronary nitroglycerin injection, which excluded coronary spasms as the causative factor (Fig. 2B). An intravascular ultrasound (IVUS) scan showed a focal soft plaque with a ruptured cap and thrombus formation (Fig. 2C). Percutaneous coronary angioplasty and stenting (Promus, $3.5 \times 18 \mathrm{~mm}$ ) were performed successfully. After coronary angioplasty, the chest pain and T-wave inversions on ECG disappeared. Anagrelide was discontinued, anti-platelet and anti-anginal agents were added to the treatment, and the patient was discharged without further cardiac symptoms.

\section{DISCUSSION}

ET is a myeloproliferative neoplasm with a chronic clinical course [1]. Patients with ET may experience thromboembolic episodes and, less frequently, hemorrhagic events. If an effective cytoreductive therapy is not introduced, acute coronary
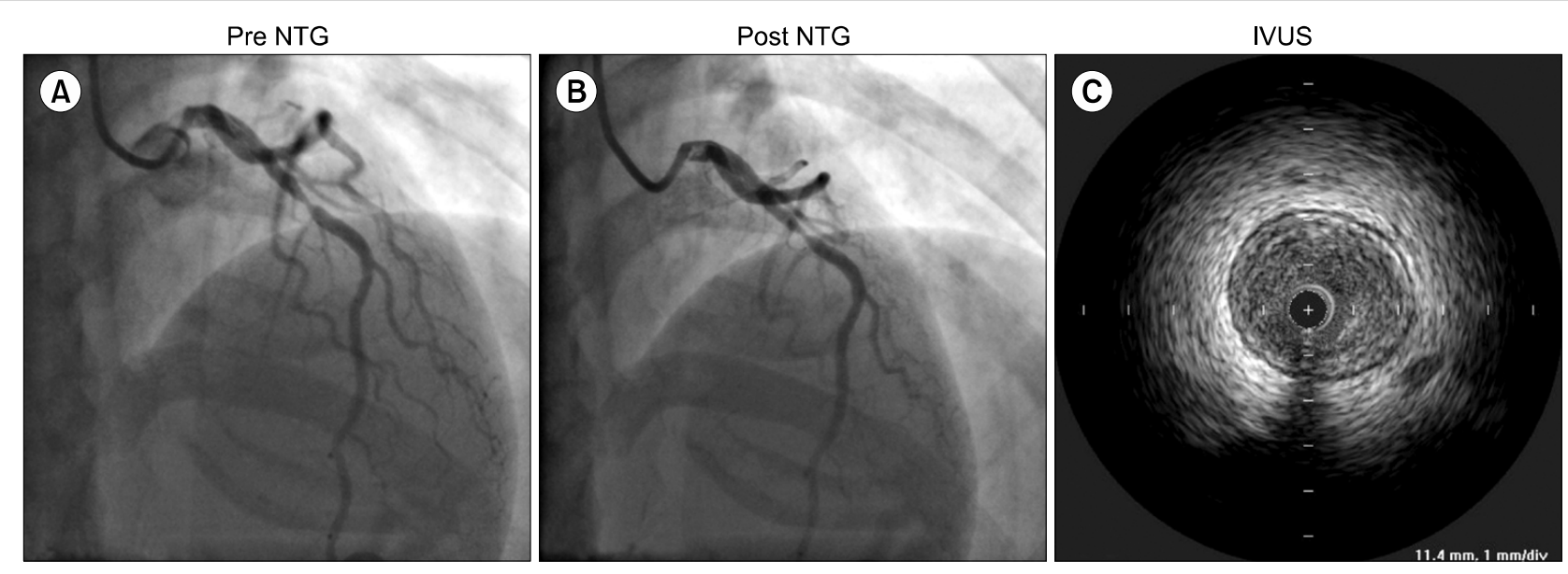

Fig. 2. Coronary angiography (CAG) and intravascular ultrasound (IVUS). (A) Left CAG before intracoronary nitroglycerin injection. (B) Left CAG after intracoronary nitroglycerin injection. (C) Plaque rupture and thrombus formation in an IVUS image. 
syndromes resulting from coronary artery involvement may develop in patients with ET. However, case reports of MIs in well-controlled cases of ET are rare, and most reports are of elderly subjects with various cardiovascular risk factors $[7,10,11]$. Our patient was a young woman with no smoking history and without hypertension, dyslipidemia, diabetes mellitus, or obesity. Although the patient had an underlying disease, ET, the disease was well controlled and she had a normal platelet count. Considering that the risk of thrombotic events decreases in patients with ET who have lower platelet counts, we tentatively suggest the possibility that anagrelide was the cause of the acute MI in this patient.

The most common side effects of anagrelide are headache, nausea, diarrhea, edema, palpitations, and an increased number of myocardial contractions [4, 9]. Potential cardiovascular adverse effects include vasospasm of the coronary arteries, congestive heart failure, arrhythmia, or acute coronary syndrome $[4,6,8]$. In addition, myocarditis, silent MI, and myocardial ischemia have been reported in young patients with ET and without cardiovascular risk factors following the administration of anagrelide [4]. Although a clear correlation between the administration of anagrelide and these events has not been well established, studies demonstrate that the adverse events disappear after anagrelide is discontinued [4]. In this patient, the segmental stenotic lesion in the mid LAD did not respond to an intracoronary nitroglyceride injection; thus, we can exclude the possibility of a vasospasm. According to the patient's coronary angiography and IVUS, a stenotic lesion within the mid LAD resulted from a plaque rupture followed by thrombus formation. Furthermore, after percutaneous coronary intervention with stent insertion, the patient's chest pain and T-wave inversions on ECG disappeared. Although MI is a rare event among ET patients treated with anagrelide, it may be an important and undesirable effect of anagrelide. The mechanism of acute coronary syndrome in patients treated with anagrelide has been reported to result from vasospasms of the coronary arteries [8], although this could not be fully elucidated in the present case. Close monitoring and prompt reporting of adverse events in clinical practice are important, as they may provide clues as to the mechanism of these undesirable side effects. It has been suggested that the majority of the side effects of anagrelide might develop within 1 month of starting therapy, and that the side effects are dose-dependent [12-14]. Although the reduction of side effects might be related to improved tolerance to treatment over time, anagrelide should be discontinued in patients who experience life-threatening adverse events, such as acute coronary syndromes $[4,8,9]$.

We treated an acute MI in a young female patient with ET who had no cardiovascular risk factors and no history of thrombotic events prior to taking anagrelide. Anagrelide is a useful drug for the treatment of ET; however, through this case we emphasize the importance of monitoring cardiovascular function both before starting therapy and during the maintenance of treatment. For young patients with ET who have a long life expectancy, the long-term benefits from discontinuing anagrelide should be weighed against the potential cardiovascular risks posed by uncontrolled ET. Finally, it is probable that combining anagrelide with anti-platelet drugs in patients with ET might be useful, as this might allow for a reduction in the dose of anagrelide, which would result in fewer side effects. For improved protection against acute coronary syndrome, it is imperative that anti-platelet drugs are added to anagrelide regimens in patients with ET who have cardiovascular risk factors.

\section{REFERENCES}

1. Tefferi A. Chronic myeloid disorders: Classification and treatment overview. Semin Hematol 2001;38(1 Suppl 2):1-4.

2. Löfvenberg E, Wahlin A. Management of polycythaemia vera, essential thrombocythaemia and myelofibrosis with hydroxyurea. Eur J Haematol 1988;41:375-81.

3. Gisslinger H, Chott A, Scheithauer W, Gilly B, Linkesch W, Ludwig $\mathrm{H}$. Interferon in essential thrombocythaemia. Br J Haematol 1991;79(Suppl 1):42-7.

4. Mazzucconi MG, Redi R, Bernasconi S, et al. A long-term study of young patients with essential thrombocythemia treated with anagrelide. Haematologica 2004;89:1306-13.

5. Tefferi A, Silverstein MN, Petitt RM, Mesa RA, Solberg LA Jr. Anagrelide as a new platelet-lowering agent in essential thrombocythemia: mechanism of action, efficacy, toxicity, current indications. Semin Thromb Hemost 1997;23:379-83.

6. Proietti R, Rognoni A, Ardizzone F, Maccio S, Santagostino A, Rognoni G. Atypical Takotsubo syndrome during anagrelide therapy. J Cardiovasc Med (Hagerstown) 2009;10:546-9.

7. Bildirici U, Celikyurt U, Ural E. Essential thrombocythemia: a case of acute ST-segment elevation myocardial infarction in a young female. Clin Cardiol 2009;32:104-5.

8. Lin GM, Chao TY, Wang WB. Acute coronary syndromes and Anagrelide. Int J Cardiol 2007;117:e17-9.

9. Birgegård G. Long-term management of thrombocytosis in essential thrombocythaemia. Ann Hematol 2009;88:1-10.

10. Terada H, Satoh H, Uehara A. Multivessel coronary thrombosis, acute myocardial infarction, and no reflow in a patient with essential thrombocythaemia. Heart 2000;83:E10.

11. Nurkalem Z, Uslu N, Gorgulu S, Eren M. Left main coronary thrombosis with essential thrombocythemia. J Thromb Thrombolysis 2006;22:165-7.

12. Steurer M, Gastl G, Jedrzejczak WW, et al. Anagrelide for thrombocytosis in myeloproliferative disorders: a prospective study to assess efficacy and adverse event profile. Cancer 2004;101:223946.

13. Penninga E, Jensen BA, Hansen PB, et al. Anagrelide treatment in 52 patients with chronic myeloproliferative diseases. Clin Lab Haematol 2004;26:335-40.

14. Birgegård G, Björkholm M, Kutti J, et al. Adverse effects and benefits of two years of anagrelide treatment for thrombocythemia in chronic myeloproliferative disorders. Haematologica 2004;89: 520-7. 